\title{
CO-INFECTION OF BRUCELLA AND DENGUE VIRUS
}

\section{DEVARATI DUTTA, PRABIR KUILA, DEBASMITA CHATTERJEE, SATADAL DAS*}

Department of Microbiology, Brucella Research Laboratory, Peerless Hospital and B. K. Roy Research Centre, Kolkata, West Bengal, India. Email: drsatdas@hotmail.com

Received: 20 September 2016, Revised and Accepted: 14 October 2016

ABSTRACT

Objective: Brucellosis is an important zoonotic infection having a worldwide distribution. In South East Asian developing countries like India, brucellosis is yet well-known but often neglected disease. Although dengue fever has a global distribution, South East Asian region together with the Western Pacific region represents $75 \%$ of the current global disease burden. Frequent dengue fever outbreaks have been encountered in different parts of India. Both brucellosis and dengue fever may present with many nonspecific symptoms. This study was designed to identify the trend of their co-infection.

Methods: The study was carried out from February 2015 to July 2016 in Eastern India. Blood samples collected from clinically suspected dengue fever cases were confirmed serologically by dengue specific NS1, IgM, and IgG capture ELISA methods. The confirmed dengue samples were further subjected to Brucella specific serological and molecular investigations.

Results: Out of 37 serologically confirmed dengue cases, 8 (21.6\%) showed co-infection of brucellosis and dengue fever. Female predominance was observed among the co-infection cases. Most of these cases belonged to the age group 21-40 years and resided in and around Kolkata.

Conclusion: This study portrays the simultaneous acquisition of these two pathogens in a few patients. Co-infection of these two pathogens, while feasible, has not been accounted previously in this part of India. Thus, this report highlights the significance of studying the possibility of occurrence of dual infection of these two diseases and its outcome in patients with febrile illness thereby providing future scope of preventing case fatalities.

Keywords: Brucellosis, Dengue, Co-infection, ELISA, Polymerase chain reaction.

(C) 2017 The Authors. Published by Innovare Academic Sciences Pvt Ltd. This is an open access article under the CC BY license (http://creativecommons. org/licenses/bv/4. 0// DOI: http://dx.doi.org/10.22159/aipcr.2017.v10i1.15259

\section{INTRODUCTION}

Brucellosis is a multisystem zoonotic disease with a broad spectrum of clinical manifestations. The disease can be transmitted through consumption of unpasteurized milk and other dairy products such as cheese, in close contact with infected animals, inhaling infected aerosols, through conjunctiva or through cuts on the skin [1]. Records of human brucellosis revealed human to human transmission are rare and are not accounted much [2]. This Gram-negative coccobacillus organism has several species among which four are significant pathogens in humans. These include Brucella melitensis, Brucella abortus, Brucella suis, and B. canis [3]. Infections due to B. melitensis are higher in humans followed by B. abortus and B. suis [4]. Brucellosis may occur as an acute or chronic disease [5]. Common presenting symptoms of this disease are undulant fever, joint and muscle pain, headache, night sweat, fatigue, and weight loss [6]. Dengue fever is a mosquito-borne tropical disease caused by the dengue virus (DENV). It is an RNA virus belonging to the genus Flavivirus. It has mainly four serotypes - DENV1, DENV2, DENV3, and DENV4. These arboviruses are transmitted by the mosquitoes - Aedes aegypti and Aedes albopictus [7]. Symptoms include high fever, headache, vomiting, abdominal pain, muscle and joint pains, and a characteristic skin rash. Brucellosis shares common clinical parameters with dengue fever in endemic areas which make their differentiation a diagnostic challenge to the clinicians. Previous reports of co-infection of these two pathogens from India are absent. The present study has been conducted to describe the real picture of co-infection of Brucella and DENV in Eastern part of India.

\section{METHODS}

The study period was 18 months from February 2015 to July 2016. Blood samples from clinically suspected dengue cases attending the outpatient (medicine) department and also from patients admitted to a tertiary care hospital in Kolkata, located in Eastern part of India, were collected. This study was approved by the Institutional Ethical Committee. Consent and detailed history of all patients were documented. Serum was separated from each blood sample using the standard protocols and was tested for detection of dengue NS1 antigen, dengue specific IgM/IgG antibody by ELISA test kits (J. Mitra \& Co. Pvt. Ltd., New Delhi, India). Serologically confirmed dengue positive sera samples (IgM antibody positive was the main criterion) were further tested by Brucella specific serological techniques such as standard tube agglutination test (STAT; Tulip Diagnostics Pvt. Ltd., Goa, India), 2 mercaptoethanol test (2 ME; Sigma-Aldrich Chemicals Pvt. Ltd., Bengaluru, India), Rose Bengal plate test (RBPT; IAHVB, Bengaluru, India), IgM and IgG ELISA (Immunolab $\mathrm{GmbH}$, Germany), and also by Brucella genus-specific molecular test like simplex polymerase chain reaction (PCR) carried out in a thermal cycler (Prime, UK). For PCR test, DNA was extracted from the serum samples using the spin column technique and following the instructions provided in the QIAmp DNA Blood Mini Kit (Qiagen, Germany). By this PCR technique, the serum samples were screened for the presence of the bcsp gene which is present in all Brucella spp.

\section{RESULTS}

Among the 37 serologically positive dengue fever cases, eight (21.6\%) were found positive for brucellosis. The details of the co-infection cases (Table 1) revealed the predominance of females among the affected cases. Furthermore, the common affected age group was 21-40 years and majority of the patients resided in and around Kolkata city. The co-infection cases were mostly obtained in 2015 in the month of September followed by the month of November. The data (Table 2) showed the results of the tests performed for the cases thought to be 
co-infected by Brucella and DENV during the study period. Information regarding other investigations done for the dual pathogen affected patients is discussed below.

Case 1: After admission on the $2^{\text {nd }}$ day, the patient showed no reactivity to dengue IgM and IgG ELISA tests. Widal test was also nonreactive. Platelet count was 110,000/cumm. Hemoglobin level was $11.9 \mathrm{~g} / \mathrm{dl}$. White blood cell (WBC) count was $2600 /$ cumm. Creatinine was $0.58 \mathrm{mg} / \mathrm{dl}$. Total bilirubin was $0.3 \mathrm{mg} / \mathrm{dl}$. Total protein was $6.5 \mathrm{~g} / \mathrm{dl}$. On the $4^{\text {th }}$ day of admission, the patient was reactive to dengue IgM and nonreactive to dengue IgG test. Further, the patient gave positive results for the Brucella specific serological tests such as STAT, 2 ME, RBPT, and IgM ELISA.

Case 2: On the day of admission, the patient was reactive to dengue IgM test and nonreactive to dengue IgG test. Dual malaria antigen was absent. Platelet count was 135,000/cumm. Hemoglobin was $15.7 \mathrm{~g} / \mathrm{dl}$. C-reactive protein (CRP) and alanine transaminase (ALT) were $<6.0 \mathrm{mg} / \mathrm{L}$ and $260 \mathrm{U} / \mathrm{L}$, respectively. WBC count was $3280 /$ cumm. Creatinine was $0.71 \mathrm{mg} / \mathrm{dl}$. Prothrombin time was 12.2 seconds. The patient gave positive results for Brucella specific STAT, $2 \mathrm{ME}$, and RBPT tests.

Case 3: On $2^{\text {nd }}$ day after admission, the patient was reactive to both dengue IgM and IgG tests. Malaria parasite was not found. Platelet count was $60,000 /$ cumm. Hemoglobin was $12.5 \mathrm{~g} / \mathrm{dl}$. WBC count was $4010 /$ cumm. Creatinine and urea were $0.73 \mathrm{mg} / \mathrm{dl}$ and $21 \mathrm{mg} / \mathrm{dl}$, respectively. The patient showed positivity in Brucella specific STAT, 2 $\mathrm{ME}$, and RBPT tests.

Case 4: On the day of admission, the patient was found nonreactive to both dengue IgM and IgG ELISA tests. The patient was found reactive to dengue NS1 antigen on the same day. Dual malaria antigen was absent. Platelet count was 66,000/cumm. Hemoglobin was $10.9 \mathrm{~g} / \mathrm{dl}$. WBC count was 2210/cumm. CRP, ALT, and creatinine were $14.8 \mathrm{mg} / \mathrm{L}$, $33 \mathrm{U} / \mathrm{L}$, and $0.63 \mathrm{mg} / \mathrm{dl}$, respectively. On the $4^{\text {th }}$ day after admission, the patient was found reactive to both dengue IgM and IgG antibody tests. The patient showed positivity in Brucella specific STAT, 2 ME, and RBPT tests.

Case 5: On the day of admission, the patient was found reactive to dengue IgM ELISA and nonreactive to dengue IgG ELISA. Platelet count was 194,000/cumm. The patient gave positive results for Brucella specific STAT, $2 \mathrm{ME}$, RBPT, and IgM ELISA tests.

Table 1: Details of Brucella and dengue co-infection patients

\begin{tabular}{llll}
\hline Case Number & Sex & Age (years) & Location \\
\hline 1 & Female & 37 & 24 Parganas (S) \\
2 & Male & 26 & Midnapore (E) \\
3 & Female & 65 & Kolkata \\
4 & Female & 30 & Kolkata \\
5 & Female & 26 & Kolkata \\
6 & Male & 58 & 24 Parganas (S) \\
7 & Male & 25 & Jharkhand \\
8 & Female & 12 & Howrah \\
\hline
\end{tabular}

Case 6: On the $3^{\text {rd }}$ day after admission, the patient was found reactive to both dengue IgM and IgG tests. Platelet count was 120,000/cumm. Hemoglobin was $16.0 \mathrm{~g} / \mathrm{dl}$. WBC count was $4230 / \mathrm{cumm}$. Creatinine and urea were $0.85 \mathrm{mg} / \mathrm{dl}$ and $9.0 \mathrm{mg} / \mathrm{dl}$, respectively. The patient showed positive results for Brucella specific serological tests such as STAT, $2 \mathrm{ME}$, RBPT, and the infection was also confirmed by Brucella genus-specific PCR test.

Case 7: On the day of admission, the patient was reactive to dengue IgM ELISA and nonreactive to dengue IgG ELISA. Hemoglobin was $13.5 \mathrm{~g} / \mathrm{dl}$. WBC count was $9380 /$ cumm. CRP and creatinine were $210 \mathrm{mg} / \mathrm{L}$ and $0.92 \mathrm{mg} / \mathrm{dl}$, respectively. Total bilirubin was $0.7 \mathrm{mg} / \mathrm{dl}$. Total protein was $6.50 \mathrm{~g} / \mathrm{dl}$. Brucella specific tests such as STAT, $2 \mathrm{ME}$, and RBPT were found positive for this patient.

Case 8: After admission on the $4^{\text {th }}$ day, the patient was found reactive to dengue IgM capture ELISA test and nonreactive to dengue IgG capture ELISA test. WBC count was 6250/cumm. Hemoglobin was $12.7 \mathrm{~g} / \mathrm{dl}$. Blood albumin was $4.02 \mathrm{~g} / \mathrm{dl}$. Brucella specific serological tests such as STAT, 2 ME, and IgM ELISA were found positive for this patient. Further, the disease was confirmed by the Brucella genus-specific PCR test.

Considering the positive serological test for dengue fever (Table 2) and also the positive serological and molecular tests for brucellosis (Table 2), these patients were diagnosed as having co-infection of both the pathogens. Thus, during the study tenure, we recorded eight confirmed cases of co-infection of Brucella and DENV.

\section{DISCUSSION}

DENV is responsible for the most frequentarthropod-borneviral infection worldwide. In India, the occurrence of first epidemic of this disease was reported from Kolkata, in 1963, which was followed by other states [8]. Brucellosis is one of the most frequent bacterial zoonosis throughout the world. In India, the prevalence of human brucellosis was first described almost a century back, but the actual disease burden is not established and the published literature shows variability. Simultaneously to the growth of the incidence of dengue fever, cases of bacterial co-infection in dengue have been increasingly reported. However, reports of co-infection of Brucella and DENV are very few. Till now, two studies showed this kind of co-infection both of which were from Saudi Arabia. Among these studies, the first was reported in 2006 [9] and the other was recorded in 2010 [10]. From India, no such occurrence of this sort of dual infection is documented so far. Therefore, this study was done to recognize the simultaneous Brucella infection either in acute or chronic stage among the dengue fever affected patients who were admitted or attended the clinic of a tertiary care hospital in Eastern India. Both brucellosis and dengue fever present as acute febrile illness with other nonspecific symptoms thus creating a diagnostic dilemma for the clinicians. A high index of clinical suspicion coupled with appropriate diagnostic tests is essential to detect this kind of co-infection at an early stage. Diagnosis of both these diseases usually depends on serological techniques because of its widespread availability and ease of use. Other definitive diagnostic technique such as PCR for detection of Brucella spp. was also carried out in this study. During the study period, we found eight cases of Brucella and DENV co-infection and it can be said that this type of disease incidence is not only the first documentation from this country

Table 2: Diagnostic tests done for co-infection cases

\begin{tabular}{|c|c|c|c|c|c|c|c|c|c|}
\hline Case Number & Dengue NS1 & Dengue IgM & Dengue IgG & STAT & $2 \mathrm{ME}$ & RBPT & Brucella IgM & Brucella IgG & PCR \\
\hline 1 & - & + & - & + & + & + & + & - & - \\
\hline 2 & - & + & - & + & + & + & - & - & - \\
\hline 3 & - & + & + & + & + & + & - & - & - \\
\hline 4 & + & + & + & + & + & + & - & - & - \\
\hline 5 & - & + & - & + & + & + & + & - & - \\
\hline 6 & - & - & + & + & + & + & - & - & + \\
\hline 7 & - & + & - & + & + & + & - & - & - \\
\hline
\end{tabular}

STAT: Standard tube agglutination test, 2 ME: 2 mercaptoethanol, RBPT: Rose Bengal plate test, PCR: Polymerase chain reaction 
but also it is much higher in number than reported in previous studies. Hence, proper monitoring of this co-infection in this part of India can lead to early recognition and implementation of definitive treatment to patients affected by this dual infection. It was noticed (Table 2) that the majority of the dengue fever affected patients had DENV IgM antibodies which is a marker of primary dengue infection and can be detected from as early as 3 days to 60 days of infection. One patient had both DENV IgM and IgG antibodies denoting secondary dengue infection as she was also affected by Brucella. DENV specific IgG antibodies appear at about $7^{\text {th }}$ day, reaches peak within 2-3 weeks and persist for life [11]. Earlier study reported that in secondary dengue infection IgG levels remain at higher levels for about 30-40 days than observed in primary or past dengue infection, and mostly, these cases are also accompanied by elevation of IgM antibody titer [12]. Another patient showed positivity to all dengue specific serological tests done in this study. On the day of admission, the patient was reactive to dengue NS1 antigen ELISA test which can be detected from as early as 1-day post onset syndrome and remains positive till 18 days [13]. On the $4^{\text {th }}$ day after admission, dengue IgM and IgG antibodies were found in that female patient. All these cases of dengue fever showed simultaneous positivity in one or more Brucella specific tests conducted in the study. It was also observed that among these patients, three patients had both Brucella IgM antibodies and DENV IgM antibodies indicating that both these diseases were in their acute stage. An explanation for this phenomenon can be the possible cross-reactivity between Brucella and DENV. However, earlier reports do not reveal such kind of cross-reactivity till date. Cross-reactivity of Brucella with other bacteria are reported [14] but no reports regarding cross-reactivity with viruses particularly the Flavivirus group has been published. On the other hand, prior reports showed that the antibodies from the sera of dengue fever affected patients can cross-react with the antibodies of other Flavivirus like Japanese encephalitis and yellow fever viruses [15]. Therefore, these patients can be considered as cases of co-infection of these two pathogens and not cross-reactivity among these pathogens. Although brucellosis generally proceeds toward chronicity [3], it was found that in this study among the dual pathogen infected patients, none of them suffered from chronic brucellosis as Brucella IgG ELISA was negative in all cases.

\section{CONCLUSION}

In this study, the simultaneous serological and molecular biological documentation of these two pathogens are described suggesting dual infection in few patients with febrile illness. This is the first report of this kind from the Eastern part of India. To conclude, this study emphasizes the need of continuous monitoring to identify co-infection of Brucella and DENV. Furthermore, it can be said that this type of study will enable the clinicians the scope to recognize the signs early and start proper treatment which will prevent future worsening outcome in mortality and morbidity rates.

\section{ACKNOWLEDGMENT}

We would like to acknowledge the grant received from the Government of India (Department of Biotechnology, DBT Sanction Order No. 102/IFD/SAN/3141/2012-2013) for giving us the opportunity to conduct this study. We would also like to give special thanks to all those people attached to Brucella Research Laboratory and Peerless Hospital and B. K. Roy Research Centre whose help and dedication made this work possible.

\section{REFERENCES}

1. Mondal I, Sanyal S, Das S. Coagulation profile in patients suffering from acute brucellosis. Asian J Pharm Clin Res 2013;6:179-80.

2. Saha S, Gupta D, Das S. Autoimmune changes in human brucellosis. Int J Biopharm 2013;4:131-4.

3. Pappas G, Akritidis N, Bosilkovski M, Tsianos E. Brucellosis. N Engl J Med 2005;352(22):2325-36

4. Chatterjee D, Halder D, Chakraborty U, Dutta D, Kuila P, Das S. Antimicrobial activities of pharmacological and antioxidant compounds of some edible mushrooms against Brucella spp. Int J Pharm Sci Res 2016. [In Press].

5. Sen A, Banerjee P, Dutta D, Pal MK, Ray A, Das S. Brucellar uveitis in Eastern India. Asian J Pharm Clin Res 2016. [In Press].

6. Atluri VL, Xavica MN, De Jong MF, Den Hartigh AB, Tsolis RE. Interactions of the human pathogenic Brucella species with their hosts. Annu Rev Microbiol 2011;65:523-41.

7. Lambrechts L, Scott TW, Gubler DJ. Consequences of the expanding global distribution of Aedes albopictus for dengue virus transmission. PLoS Negl Trop Dis 2010;4(5):e646.

8. Garg A, Garg J, Rao YK, Upadhay GC, Sakhuja S. Prevalence of dengue among clinically suspected febrile episodes at a teaching hospital in North India. J Infect Dis Immun 2011;3:85-9.

9. Ayyub M, Al-Juhani NR, Alfi AY, Al-Ukayli S. Brucellosis and dengue fever - a co-infection or cross reactivity? Biomedica 2006;22:80-3.

10. Bzeizi KI, Benmousa A, Sanai FM. Coincidence of acute Brucella hepatitis and dengue fever or serologic cross-reactivity? Saudi J Gastroenterol 2010;16(4):299-301.

11. Vijayakumar TS, Chandy S, Sathish N, Abraham M, Abraham P, Sridharan G. Is dengue emerging as a major public health problem? Indian J Med Res 2005;121(2):100-7.

12. Innis $B L$, Nisalak A, Nimmannitya $S$, Kusalerdchariya $S$, Chongswasdi V, Suntayakorn S, et al. An enzyme-linked immunosorbent assay to characterize dengue infections where dengue and Japanese encephalitis co-circulate. Am J Trop Med Hyg 1989;40(4):418-27.

13. Laboratory Guidance and Diagnostic Testing. Centers for Disease Control and Prevention. Available from: http://www.cdc.gov/dengue/ clinicallab/laboratory.html. [Last accessed on $2013 \mathrm{Jul} 20$ ].

14. Chart H, Okubadejo OA, Rowe B. The serological relationship between Escherichia coli $\mathrm{O} 157$ and Yersinia enterocolitica $\mathrm{O} 9$ using sera from patients with brucellosis. Epidemiol Infect 1992;108(1):77-85.

15. Makino Y, Tadano M, Saito M, Maneekarn N, Sittisombut N, Sirisanthana V, et al. Studies on serological cross-reaction in sequential Flavivirus infections. Microbiol Immunol 1994;38(12):951-5. 